\title{
A Fear State Judgement System for Alleviating Fear of Heights Gradually in VR
}

\author{
Iku Kitanosono $^{1+}$, Toshiyuki Haramaki ${ }^{2}$ and Hiroaki Nishino ${ }^{3}$ \\ ${ }^{1}$ Graduate School of Engineering, Oita University, Japan \\ ${ }^{2,3}$ Faculty of Science and Technology, Oita University, Japan
}

\begin{abstract}
VR has been expanding into various fields in recent years. The medical field is no exception to this trend. For example, VR technology is expected to be used to treat acrophobia, the most common phobia. There are many patients with acrophobia, and many of them have difficulties in their daily lives. One way to solve this problem is to treat acrophobia with VR. However, VR treatment is a big problem in the treatment of phobia because there are many individual differences. In this study, we propose a system that can acquire the fear state of an individual in real-time by vital sensing and can treat phobia of altitude gradually adjusted to the individual using a placebo effect.
\end{abstract}

Keywords: acrophobia, VR, medical treatment, vital sensing, placebo effect

\section{Introduction}

In recent years, VR has made inroads into various fields, and research is underway in various places. The medical field is no exception to this trend and increasingly used to treat phobias. Although it is normal for people to feel a sense of fear about their own life and death, patients with acrophobia may have negative feelings about heights, which can damage their daily lives and the people around them. Therefore, it was considered desirable to develop simulation software that can easily treat acrophobia using VR. VR systems for the treatment of acrophobia already exist, for example, in games and researches. However, all such software products are highly dependent on individual differences. From this viewpoint, we constructed a system that can provide more effective treatment for fear of heights after an approach to fill in individual differences using vital sensing and placebo effects. The development was carried out by dividing it into the VR system which treats fear of heights using the placebo effect and the system which can judge the fear condition of the users.

\section{Related Works}

We have developed a VR system to treat acrophobia using the placebo effect [1]. The objective is to treat light acrophobia. A user who wants to treat acrophobia writes a consent form to immerse into this system. Then, a user equips a VR device and board an elevator to the room which is located in high place. First, the user experiences the second floor and go upper gradually. We established the transparent level as terms to clear each floor. Transparent level means a method to make users understood the room locates high places gradually by experiencing level 1 to 3 of transparent level. Level 1 transparent window frame and cell, level 2 transparent wall, and level 3 transparent floor. This is also a placebo effect. This system defined that placebo effect means the thing which users get used to high places gradually, so user clear each transparent level and misunderstand that he is in high places visually.

\footnotetext{
${ }^{+}$Corresponding author. Tel.: + 81 070-2667-6516
}

E-mail address: v18e3009@oita-u.ac.jp 
From the result of the experience, it is effective to get used to high places gradually, but it is weak the effect to use the placebo effect. Therefore, we propose a more effective method of improving phobia by combining the fear state judgment system described in this paper with the placebo effect.

\section{Methodology}

\subsection{Summary}

As described in 2, a VR system for treat fear of heights needs to determine the fear state. So, to indicate the fear state visually, this study determined the fear state by mapping the acquired vital data of the user onto Russell's emotional model [2]. Various famous models have been proposed so far. However, since Ekman's basic facial expression [3] is for facial expressions, it is inappropriate for this system where most of the face is hidden in VR. Besides, the method of mapping the vital sensed data seems to be difficult in the circle of the feeling model put forward by Plutchik. Therefore, we used Russell's ring model, which consists of 2 opposing axes. Fig. 1 shows Russell's emotional model, that contains of the x-axis for comfort and discomfort and the $y$-axis for arousal and sleepiness. The state of fear is determined when the patient is in an arousal and discomfort state located in the second quadrant.

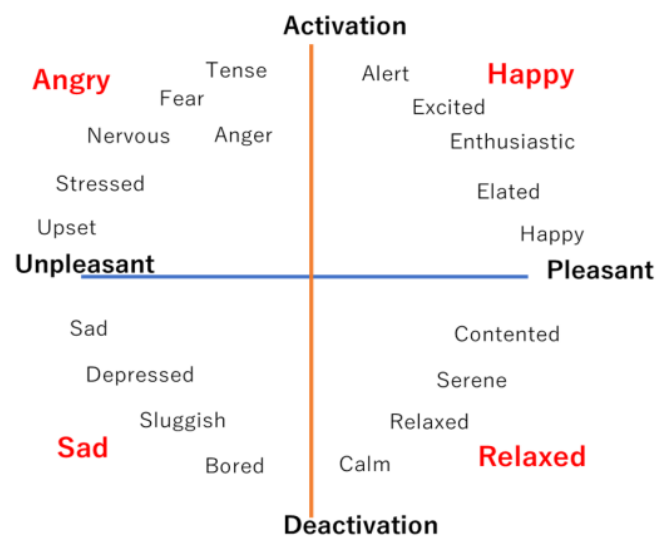

Fig. 1: Russell's emotional model

\subsection{Mapping on Russell's Emotional Model}

To mapping on Russell's emotional model, the Pleasant/Unpleasant axis is acquired by EEG, and the Activation/Deactivation axis was acquired by ECG. It is possible to get relaxation or concentration data from EEG. In addition, it is known that drowsiness also appears in ECG.

The fear or tension state is in the second quadrant on Russell's emotional model and it is necessary to detect an Unpleasant state on the x-axis and Activation state on the y-axis. Since the Unpleasant state is acquired from the EEG, the degree of relaxation can locate as the x-axis component. Moreover, regarding the Activation state, it is necessary to measure the activity level of the sympathetic nerve. The sympathetic nerve is a nerve in a tension mode that is active during the day, and the parasympathetic nerve is a nerve in a relaxation mode that is predominantly active at night. For this reason, the sympathetic nerve becomes active in the awake state, and the parasympathetic nerve becomes active in the sleepy state.

The ECG has a period and the part with the largest amplitude within the period is called R. The interval between $\mathrm{R}$ and $\mathrm{R}$ is called the R-R interval (RRI).

$$
\text { - } r M S S D=\sqrt{\frac{1}{N-1} \sum_{i=1}^{N-1}\left(x_{i}-x_{i+1}\right)^{2}}
$$

rMSSD is a parameter representing the mean square root of the square of the difference of RRI and representing the activity state of the parasympathetic nerve.

$$
\text { - } S D N N=\sqrt{\frac{1}{N} \sum_{i=1}^{N}\left(x_{i}-\bar{x}\right)^{2}}
$$

SDNN is the standard deviation of RRI. This is a parameter that represents the sympathetic and parasympathetic activity states. Since this ratio can be used to determine the state of sympathetic activity, it is necessary to check whether the SDNN / rMSSD value has increased. 


\section{System Implementation}

\subsection{Mapping to the Axis Representing Pleasant/Unpleasant}

EEG is used to determine the position along the pleasant and unpleasant axis. Our system used a MindWave Mobile 2 [4] which is developed by NeuroSky Co. to acquire EEG. EEG data is sent and received via Bluetooth connection with PC. Since there was only a Python 2.7 library that could receive data from the device, we created a Python 2 program that receives the data, sends it over a socket to localhost, and then creates a Python 3.6 program that receives the data and summarizes it with heart rate sampling data on the y-axis. The data sent from the device contains various values such as $\alpha$ waves and round meditation, but this time we used meditation value.

\subsection{Mapping to the Axis Representing Activation/Deactivation}

The ECG was utilized to determine the position along the activation and deactivation axis. The used device was an Arduino Uno DFRobot Heart Rate Monitor Sensor [5] with electrodes. Using Arduino language developed based on $\mathrm{C}$ language, we created a program that continuously sends RRI through this device. As a method of detecting the $\mathrm{R}$ part, the maximum value was continuously updated, and the peak value was determined when it continuously dropped to 70 percent of the maximum value, and the same was done for each period to obtain the difference. Peak value means $R$ value. And the maximum value means the maximum part for the interval. We also created a Python 3.6 program that receives RRI data and samples data as SDNN / rMSSD sent via serial communication.

\subsection{Draw to Graph on Russell's Emotional Model}

Fig. 2 shows the scatter graph which maps each acquired data. A real-time continuous data obtained in and 4.1 and 4.2 are mapped onto the graph which indicates Russell's emotional model. In the Fig. 2, the vertical and horizontal scales are different from Russell's emotional model, but they are the same. As the xaxis means 4.1 and the y-axis means 4.2. The mapping is done using the matplotlib Python library. Currently, $\mathrm{UI}$ is implemented using the scatter chart, but we plan to improve it to make it easier for users to understand.

The origin is $(\mathrm{x}$-axis $=50, \mathrm{y}$-axis $=(\mathrm{SDNN} / \mathrm{rMSSD}))$. The $\mathrm{x}$-axis origin indicates median relaxation 50 degree which has $0-100$. The $y$-axis origin is drawn with SDNN / rMSSD value excluding the user's outlier immediately after the system is started as the reference value. When the $\mathrm{x}$-axis component becomes a value of 50 or less, it indicates an Unpleasant state, so that it is located on the quadrant of $x<=50$ in the graph. Further, when the value of the y-axis component is equal to or less than 50, it indicates an uncomfortable state, and therefore, the graph is positioned in the quadrant of $x<=0$. When it is satisfied with the above two conditions, a value located in the second quadrant is obtained.

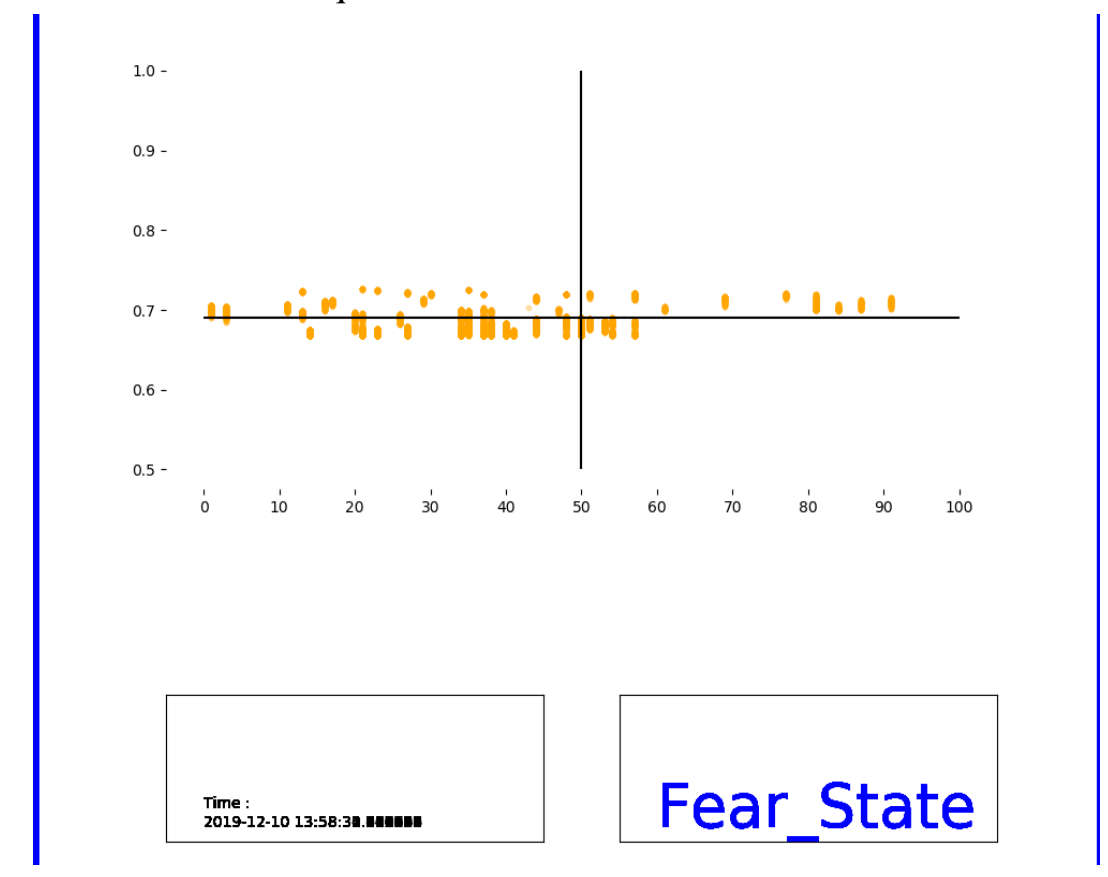

Fig. 2: This system's UI for drawing Scatter Graph which indicates Russell's Emotional Model 


\section{Preliminary Experiment}

\subsection{Outline of the Experiment}

We experimented to watch a movie which felt fear, because this system is made for judging fear state. It is necessary to acquire a fear state that the individual does not perceive, so it is necessary to perform a quantitative evaluation instead of a qualitative evaluation that depends on the subject's subjectiveness. Therefore, it is necessary to use an emotion measurement device different from this system for quantitative evaluation. We used the system called "Affedex" which was an AI to recognized human emotions. Affedex is developed by CAC company. In this case, we used the free Android app "Affedex me", because we needed to conduct experiments to evaluate the accuracy. We would like to consider the results of experiments conducted using the system for overcoming acrophobia, but since Affedex me is a system that infers emotions from the user's facial expressions, VR that covers the entire face not compatible with. Therefore, we asked the participants to experience the video. After this experiment, we wanted to have highaltitude phobia patients experience high-altitude videos and evaluate them.

\subsection{The Environment of the Experiment}

Three students wearing Mind Wave Mobile 2 and Arduino Uno DFRobot Heart Rate Monitor Sensor watched a 9-minutes horror video which includes elements that surprise you. The fear state of the student while watching the fear video is saved with the time data and all the ECG data and EEG data while watching the fear image are saved in a CSV file.

Emotions are estimated using Affedex me from the expression of the subject while watching a video. In order to perform quantitative evaluation, the results of this system and Affedex me are compared and evaluated.

\subsection{Result of the Experiment}

Fig. 3-5 shows the result of fear state detected from User A-C. The arrow at the side of "Horror Video" shows the time of the video. This system shows the fear state of User A to C which detects by this system. Affedex me shows the fear state of User A to $\mathrm{C}$ which detects by Affedex me system.

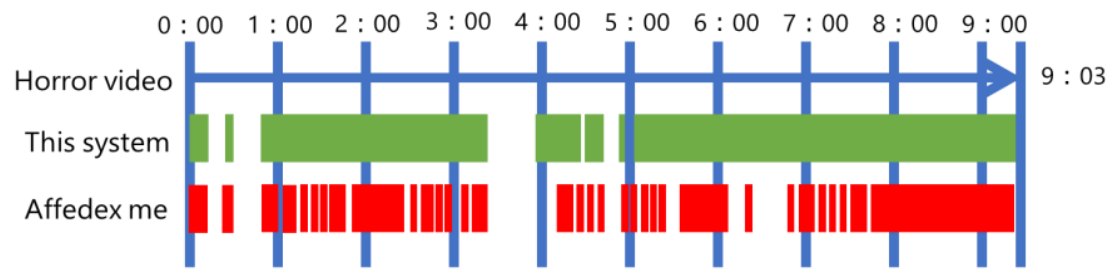

Fig. 3: User A's fear state compared to Affedex me

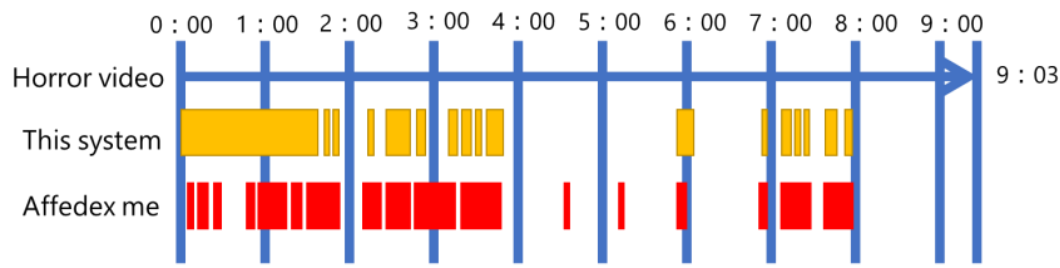

Fig. 4: User B's fear state compared to Affedex me

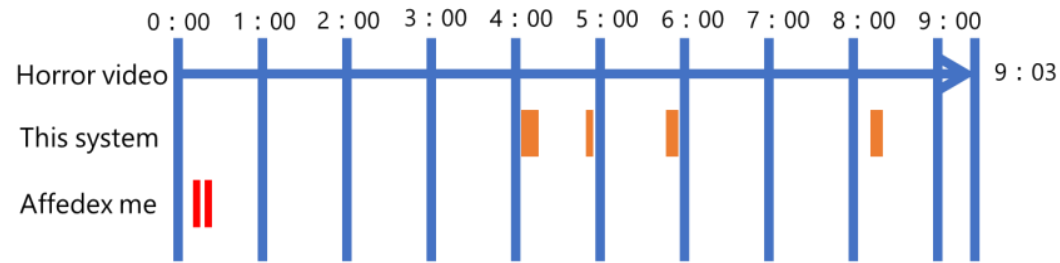

Fig. 5: User C's fear state compared to Affedex me 


\subsection{Consideration}

From the results of Fig. 3 and 4, it was confirmed that the fear states of the users A and B were normally acquired. However, as a result, Affedex me can detect the state of fear in more detail, so it is necessary to consider it as a future improvement. From the results of Fig. 5, a fear state that did not match at all was detected. Judging from the answer that the user $\mathrm{C}$ did not feel any fear when he/she checked with the user $\mathrm{C}$ after the experiment, it is considered that he/she erroneously recognized the fluctuation of vital data such as the movement by the device as the detection. In the future, I would like to be able to acquire data stably even when it operates by re-examining devices.

\section{Conclusion}

This paper proposed a fear state acquisition system for more effective treatment of acrophobia with VR. Then, we devised and implemented the algorithm, and conducted experiments to confirm and improve the accuracy of the fear state of this system. In the future, this system will be able to predict the state of fear and judge with high accuracy by introducing RNN using deep learning. Prediction reduces the mental and physical burden and allows for more gradual treatment. We also think that if we can predict emotions, we can introduce not only acrophobia but also various system.

\section{Acknowledgements}

We would like to express my appreciation to Mr. Gyohten who assisted the submission to this conference.

\section{References}

[1] Iku Kitanosono, Toshiyuki Haramaki, Tsuneo Kagawa, Hiroaki Nishino: A VR System for Alleviating a Fear of Heights Based on Vital Sensing and Placebo Effect. International Conference on Network-Based Information Systems (NBiS) 2019: Advances in Networked-based Information Systems pp 62-72

[2] James A. Russell. A circumplex model of affect. Journal of Personality and Social Psychology. Vol. 36, pp. $1161 \sim 1178.1980$

[3] P. Ekman and W.V. Friesen : Facial Action Coding System, Consulting Psychologist Press. 1977.

[4] NeuroSky MindWave Mobile2(https://www.neurosky.jp/mindwave-mobile2/)

[5] DFRobot Heart Rate Monitor Sensor(https://wiki.dfrobot.com/Heart_Rate_Monitor_Sensor_SKU_SEN0213) 